\title{
SUBACUTE NECROTIZING ENCEPHALOMYELOPATHY IN AN INFANT
}

\author{
BY \\ DENIS LEIGH \\ From the Department of Neuropathology, Institute of Psychiatry, Maudsley Hospital, London
}

The following case appears to be unique in that no references to a similar condition can be found in the literature. In addition it merits consideration as an example of the reaction of the infant brain to disease.

\section{Case Report}

K.H., a boy, aged 7 months 3 weeks, was admitted to King's College Hospital on April 22, 1947.

Birth had been normal (birth weight $6 \frac{3}{4} \mathrm{lb}$.) as also was development until six weeks before admission. The infant was vaccinated at the age of 2 months. He was breast-fed entirely for six weeks, then complementary feeding was begun.

The baby's behaviour was normal until six weeks before admission when he stopped crying, lay very still, and slept for long periods, only waking when disturbed. He did not suck and had to be fed by spoon. Sweating was increased during this period.

He had had no specific fevers.

There was one sister aged 10. At the onset of the baby's illness this sister was at home with a cold, sneezing and coughing a good deal.

On examination it was seen that the general nutrition was good, the skin normal, and the mouth and throat healthy. There was no glandular enlargement, and no palpable enlargement of liver or spleen. There was no abnormality in heart or lungs.

The pupils were small with no reaction to light, or appreciation of light ; ophthalmoscopy showed a bilateral optic atrophy, with normal maculae and retina. The infant appeared to be deaf. No other abnormality was observed in the cranial nerves. Both upper and lower limbs were markedly spastic. Reflexes were not obtained. There were bilateral extensor responses.

Investigations. - Lumbar puncture was performed on April 22 and $10 \mathrm{ml}$. fluid removed under increased pressure. Analysis showed cells 2 per c.mm.; protein $30 \mathrm{mg}$. per $100 \mathrm{c} . \mathrm{mm}$. ; globulin, negative ; sugar present ; chloride $740 \mathrm{mg}$. per $100 \mathrm{c.mm}$. Lange curve 0111000000 ; Wassermann reáction negative.

On April 23 a radiograph of the chest showed no abnormality.
The child became rapidly worse, and on April 25 was comatose, dying in terminal hyperpyrexia later the same day.

The clinical diagnosis was obscure, but an encephalitic process was considered to be the most likely cause of the fatal illness.

Necropsy.-A necropsy was performed 69 hours after death. No abnormality was observed except in the central nervous system.

The brain weighed $740 \mathrm{~g}$. There was a severe, diffuse vascular injection of the leptomeninges. The convolutions were normal in size and appearance. In coronal sections the cerebral white matter seemed somewhat greyer than normal. The left lateral ventricle was slightly dilated. The caudate and lenticular nuclei appeared normal. Mid-thalamic sections showed areas of dark grey-brown discoloration involving the anterior nucleus, dorsomedial nucleus, nucleus submedius, zona incerta, and substantia nigra on both sides. More posteriorly the lesions were limited to the medial third of the pulvinar on either side. In the midbrain similar greyish-brown areas occupied the whole tegmentum; at the level of the inferior colliculus and upper pons the process was confined to the central region between the inferior colliculi and the trapezoid bodies. In the midpons the lesion was most marked in the region of the trigeminal motor nuclei. The upper medulla showed a most striking bilaterally symmetrical red-brown discoloration of the inferior olivary nuclei ; in the lower medulla the whole of the central grey matter was similarly affected. In the spinal cord this dark redbrown appearance was seen in the posterior columns, particularly in their medial parts. The cervical region was more affected than the thoracic, whilst the lumbar segments did not show this change.

Microscopic Findings.-Histologically all the areas which showed macroscopic change were the site of a profuse proliferation of the smaller blood vessels, capillaries, pre-capillaries, arterioles, and venules (Fig. 1). The walls of these vessels were not thickened and in none was there either thrombosis or haemorrhage. In the situations where this vascular proliferation was present, the nerve cells were often severely damaged, showing a variety of degenerative changes. 


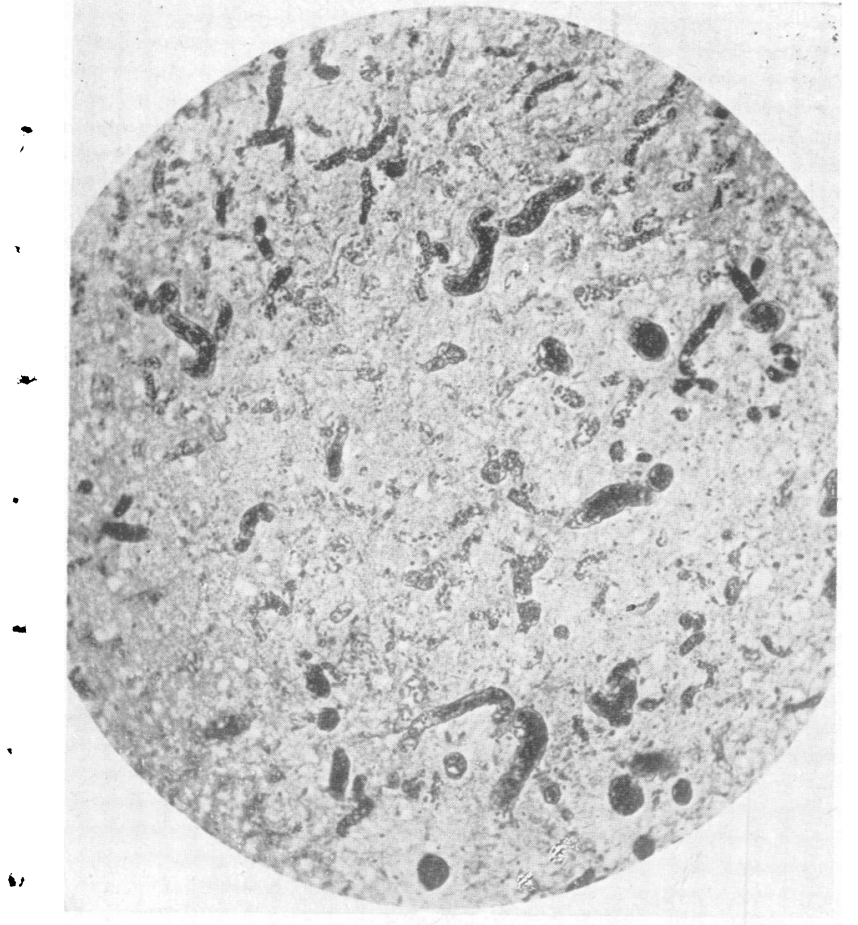

FIG. 1.

Fig. 1.-Vascular proliferation in the anterior nucleus of the thalamus. Perdrau's silver stain $\times 90$.

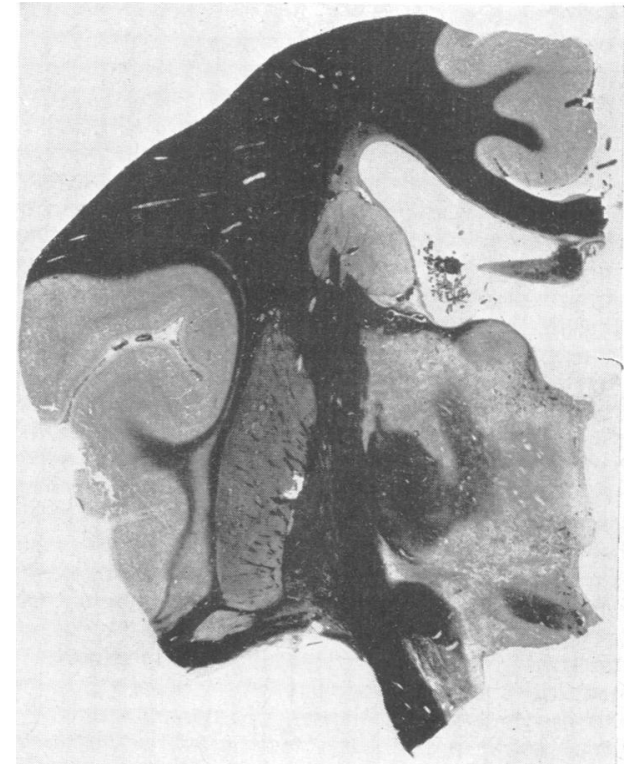

FIG. 3.

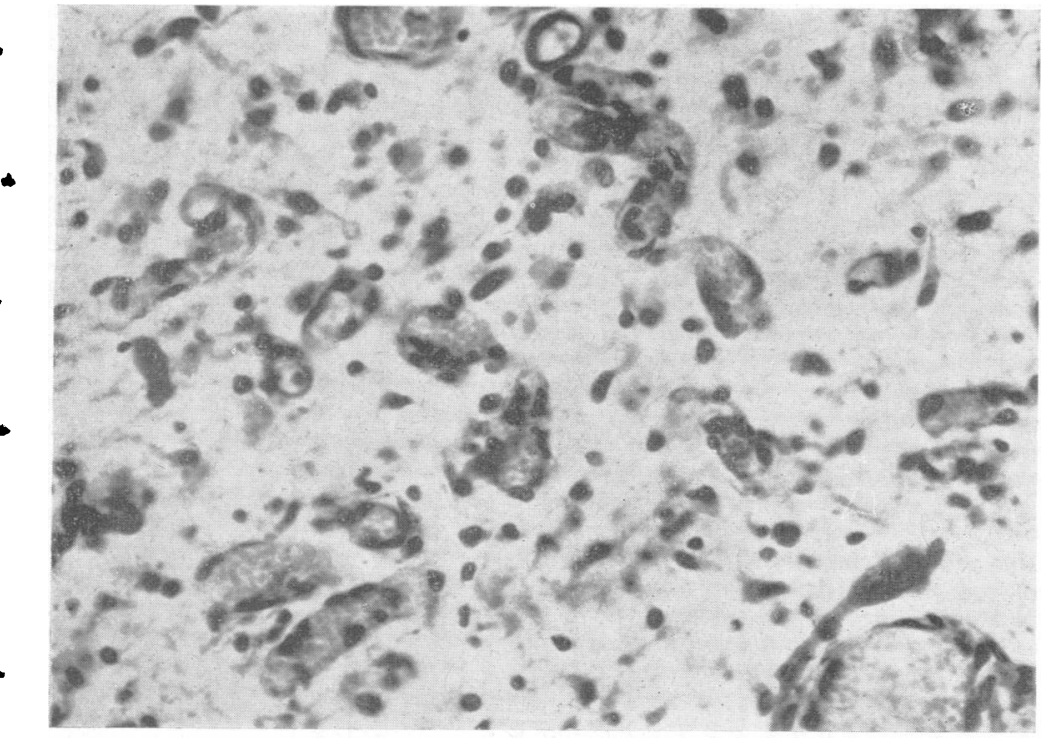

FIG. 2.-Periaqueductal grey matter of the mid-brain, showing microglial proliferation, compound granular cells, and a proliferation of capillaries and precapillaries. Nissl $\times 350$.

FIG. 3.-Thalamus at mid-dorsal level, showing destruction particularly of the anterior nucleus, nucleus magnocellularis, and the midline nuclei. Note sparing of subthalamic nucleus (corpus Luysii). Heidenhain's myelin stain $\times 2$.

FIG. 2. 


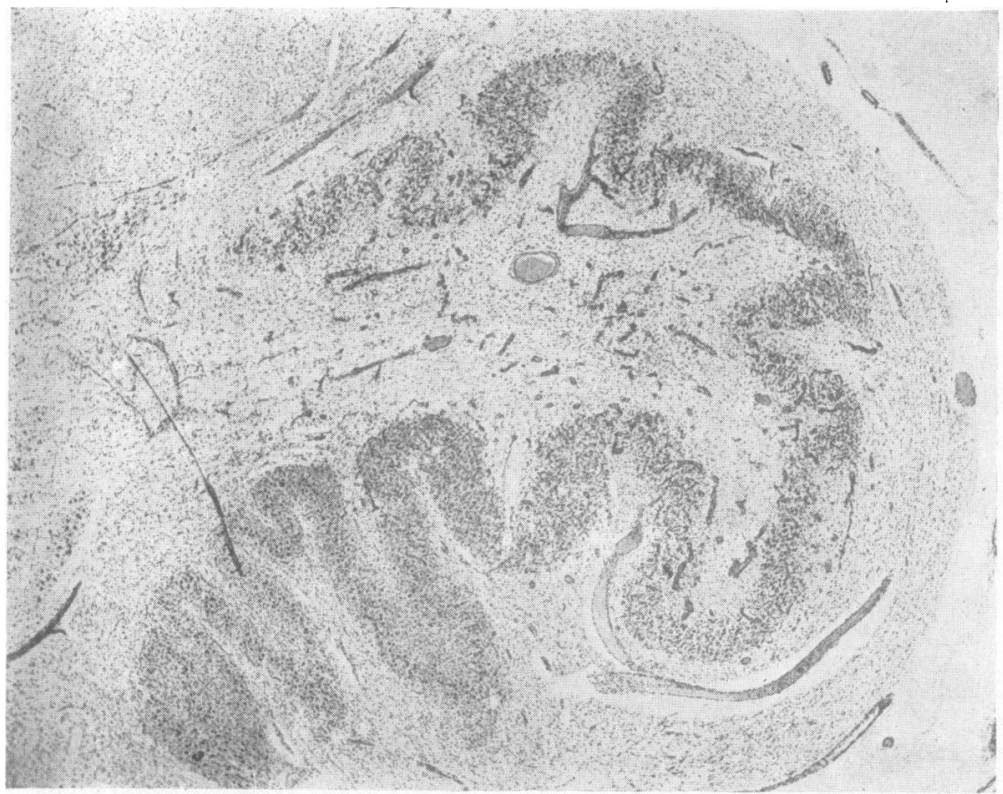

FIG. 4.-Inferior olive showing preservation of the dorso-medial portion of the nucleus. An intense glio-mesodermal reaction, with destruction of olivary nerve cells, can be seen in the dorsolateral and ventral portion of the olive. Nissl $\times 20$.

Fig. 5.-Spinal cord, fifth cervical segment. Vascular and glial proliferation in the columns of Goll. Nissl $\times 11.5$.

Fig. 6.-The same cord segment showing gliosis of the dorsal portions of the posterior columns and around the central canal. Holzer $\times 11.5$.

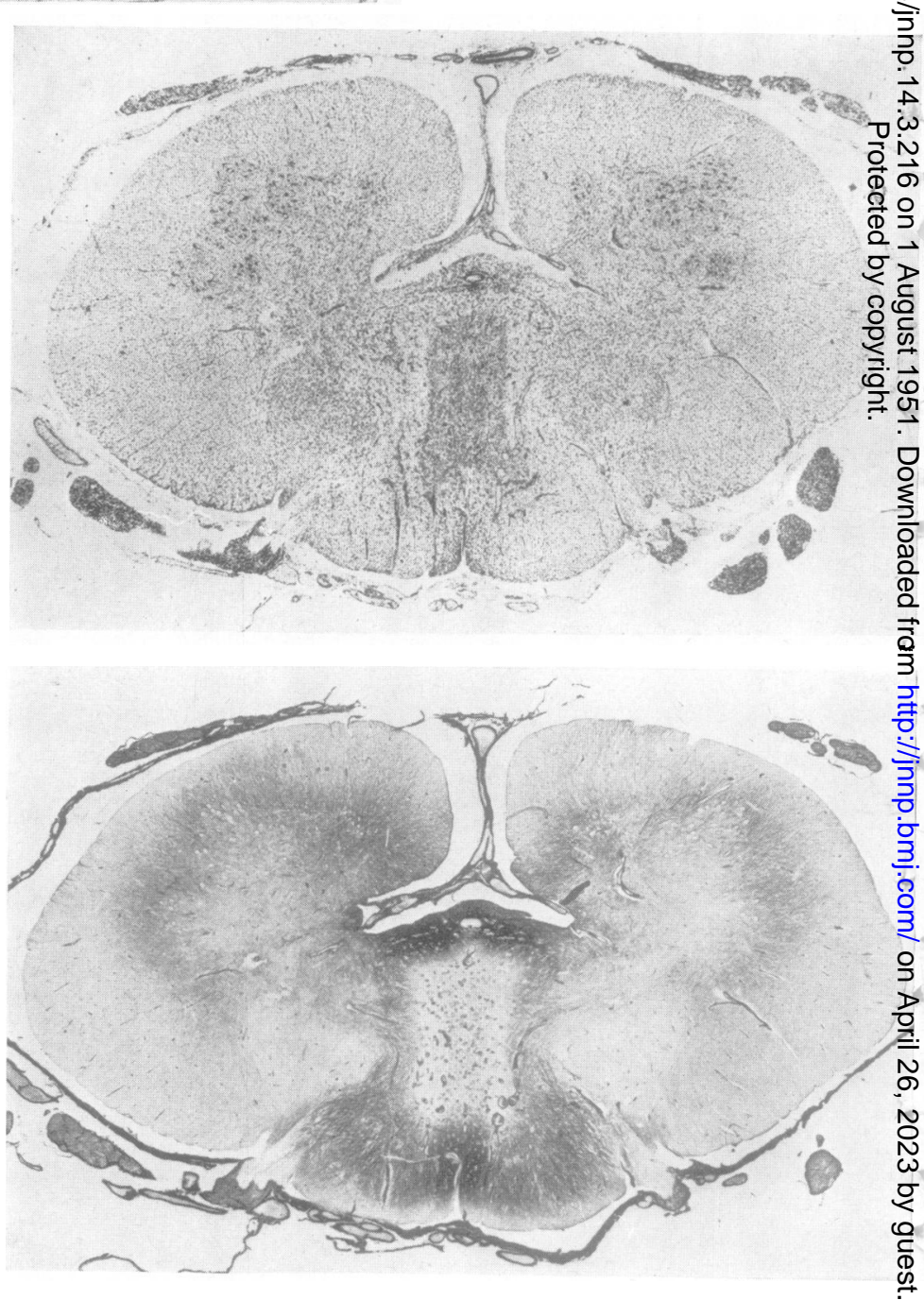


These included swelling of the cell body with central chromatolysis and nuclear swelling, general cell shrinkage, neuronophagia, and satellitosis. Normal neurons were also present, however, and the nerve cell changes were by no means as prominent as the vascular proliferation. The microglia was extremely active, all forms of transitional types being seen, up to the fully developed compound granular cell. The latter were grouped chiefly at the periphery of the lesion, often in great number. There was a considerable reduction in the number of oligodendrocytes and a general loosening of the interstitial tissue. A marked macroglial proliferation was observed both in Nissl and Holzer preparations. The general appearance of the lesion is shown in Fig. 2.

The thalamus was severely affected, particularly the anterior nucleus, nucleus magnocellularis of the dorsalis medius, and the mid-line nuclei. The parvo-cellular part of the nucleus dorsalis medius, lateralis posterior, and ventralis medalis posterior showed many of the above changes, but of less intensity, whilst the reticular and adjacent ventralis posterior lateralis, paralamellar and lateralis dorsalis nuclei, although involved, showed the mildest change. Sections stained for myelin by Heidenhain's method showed a demyelination in the regions of these nuclei (Fig. 3). The anterior thalamic radiation was also demyelinated. The optic nerves were demyelinated. Holzer preparation revealed a conspicuous gliosis in the internal capsule, in the anterior thalamic radiation, subcallosal fasciculus, optic tract, and in the septum between the fornix and the corpus callosum. There was also a subependymal gliosis of moderate degree. It was noteworthy that both the mammillary bodies, and the subthalamic body of Luys were completely unaffected. In the region of Forel's field were two bilaterally symmetrical softenings measuring 2-3 mm., with many compound granular and microglial cells and an intense proliferation of blood vessels, chiefly capillaries.

In the midbrain there was much destruction. The red nuclei were severely involved throughout their whole extent, as were the oculo-motor nuclei. The corpora quadrigemina showed patchy areas of destruction, the inferior corpus quadrigeminus being the most seriously damaged. The substantia nigra was severely affected in its lower third, the upper two-thirds of the nucleus giving a normal histological appearance. The symmetry of the lesions was remarkable, identical fields being involved on both sides. The Holzer technique showed a gliosis in the region of the red nucleus, and a heavy gliosis of the intramesencephalic course of the oculomotor nerve. There was in addition a subependymal gliosis of mild degree.

In the pons the same process was seen, occupying that portion of the pons dorsal to the trapezoid fibres and ventral to the aqueduct and upper portion of the fourth ventricle. It was remarkable that amidst this area of destruction the neurons of the trochlear nuclei stood out intact.

In the medulla a remarkable shift of the process had occurred, the inferior olives alone being affected. The dorsomedial aspects of the olives were well preserved, but the remainder of the nucleus was severely damaged.
Practically the whole of the hilus was occupied by a vascular proliferation of capillaries, pre-capillaries, arterioles, and dilated venules (Fig. 4). There was, in Holzer preparations, a moderate fibrous gliosis both of the inferior olives, and of the subependymal layer. Heidenhain preparation showed a demyelination of the white matter in the hilus of the inferior olives.

In the spinal cord the lesion was confined to the dorsal columns. In the cervical region the anterior horn cells were quite normal. The dorsal columns, particularly the gracile fasciculi, were the site of a pronounced gliomesodermal reaction (Fig. 5). Holzer sections revealed a fibrous gliosis of the dorsal portions of the posterior columns and around the central canal (Fig. 6). Heidenhain staining showed a demyelination within the affected areas. In the thoracic region the same process could be seen, but it was clearly diminishing in intensity until in the lumbar region no abnormality could anywhere be detected.

No microscopic abnormality of the meninges, meningeal blood vessels, cerebral or spinal blood vessels, apart from the changes described, was anywhere detected. The remainder of the central nervous system was within normal limits for an infant of 31 weeks.

\section{Discussion}

The extent of the lesions is represented diagrammatically in Fig. 7. The changes were bilaterally symmetrical extending from the thalamus to the thoracic cord, and their localization obviously accounted well for the clinical picture of drowsiness, blindness, deafness, and spasticity of the limbs.

Microscopically, the lesions collectively were remarkably similar in appearance, consisting of areas of softening and an intense glio-mesodermal proliferation. The vascular overgrowth was the most striking feature, but it was noticeable that elsewhere throughout the central nervous system the vessels were entirely normal. This vascular reaction was so intense as to be out of all proportion to the extent of nerve cell damage. The changes in the latter ranged from acute cell swelling and central chromatolysis to complete destruction, and the interstitial tissue (ground substance or neurophil) was loosened, or completely broken down. Of the glial elements the astrocytes had proliferated, and in certain areas had produced a well marked fibrous gliosis. The microglia was extremely active, all forms of transitional cell types being present, up to the fully developed compound granular cell.

The remarkable localization of the lesions immediately poses the problems of pathogenesis and diagnosis. The resemblance of the pathological changes to those found in subacute necrotic myelitis was impressive at first glance. However, in all cases of the latter condition described to date the process has been confined to the spinal cord, 
and to adults, usually in their fourth or fifth decades. The clinical picture of progressive amyotrophic paraplegia, with dissociated sensory changes,
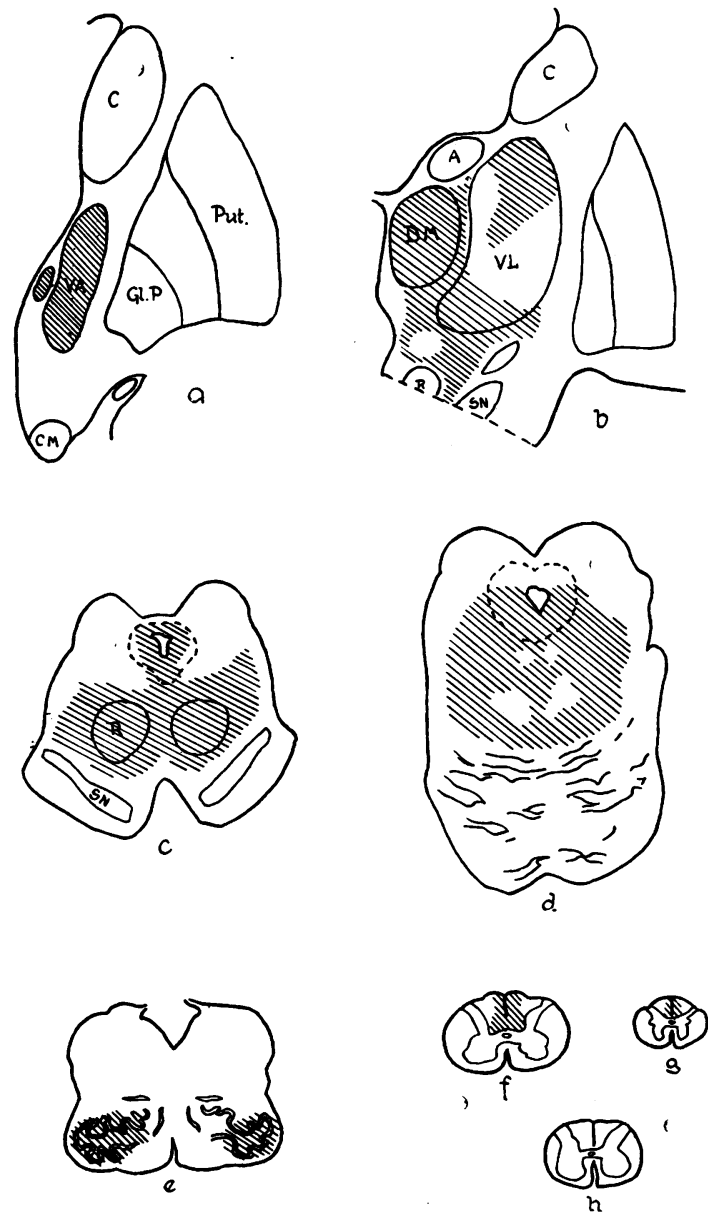

FIG. 7.-Hatched areas indicate the situations of the lesions at various coronal levels of the thalamus, brain stem, and spinal cord.

loss of tendon reflexes and of sphincter control, together with the increased cerebrospinal fluid protein, and a subacute course is very different from that presented in the patient reported here. Again, the pathological findings in this condition of a vascular overgrowth and a primary obliterative sclerosis of small intramedullary and meningeal vessels together with great thickening of the walls of the larger arteries (Greenfield and Turner, 1939) are quite distinct from the pathological appearances in the present case. Similarly, angioma racemosum venosum, which Wyburn-Mason (1943) considers to be identical with subacute necrotic myelitis, may be dismissed, as in no case has such a condition affected the nervous system above D5-D6 spinal segments.

The acute necrotizing haemorrhagic encephalopathy recently described by Adams, Cammermeyer, and Denny-Brown (1949), equine encephalitis and arsenical encephalopathy in no wise resemble this case pathologically, for in all inflammatory and haemorrhagic reactions are outspoken.

There is, however, a striking similarity between the tissue reaction here and that found in the Wernicke syndrome. There is the same vascular proliferation, the same local nerve cell changes and the same reactive proliferation of microglial and astrocytic elements. Although acute perivascular haemorrhages are common, as described by Wernicke (1881) and other authors (Spillane, 1947), nevertheless they are not invariable (Meyer, 1944). But in the Wernicke syndrome the mammillary bodies and other hypothalamic nuclei, periventricular part of medial thalamic nuclei, oculomotor nuclei, superior and inferior colliculi, periaqueductal grey matter, dorsal medulla and olives usually bear the brunt of the condition (Spillane, 1947). In this infant the mammillary bodies were not affected, and spinal cord lesions are unusual in Wernicke cases, although Bender and Schilder (1933) have described haemorrhages around Clarke's nucleus and around the lateral horns in the cervical region. The remaining lesions, however, are in situations commonly affected in the Wernicke syndrome.

The question arises as to whether this is the reaction of an immature brain to the same type of nutritional deficiency producing the Wernicke syndrome in the adult. Experimentally, the work of Ferraro and Roizin (1942) lends some weight to the suggestion that this may indeed be the case. These authors studied the neuropathological changes resulting from acute experimental inanition in the cat. Following complete deprivation of both food and water, death occurred from seven to 64 days later. In the brain two types of histological change were observed ; on the one hand nerve cell changes ranging from a diffuse chromatolysis, with nuclear pyknosis, to a widespread ischaemic cell change, and on the other hand, progressive vascular changes. These latter included a "moderate process of endarteritis" with hypertrophy and hyperplasia of endothelial cells, and a proliferation of the capillaries and pre-capillaries. In the region where this change was most marked, the astrocytes were generally larger and more numerous than usual. The localization was of interest, the cortex, subcortical areas, hypothalamus, lateral geniculate body, corpora quadrigemina, vestibular nuclei and inferior olives all being affected. These workers 
considered that the neuropathological appearances in one cat were identical with the changes found in the Wernicke syndrome. The mammillary bodies, however, were much less involved than the peri-ventricular area of the hypothalamus.

The age of the animals used in these experiments varied from kittens of six weeks to adult cats ; the type of pathological change did not differ in either group. The younger animals, however, were less resistant and died much sooner than the older animals.

Focal lesions with a similar glio-mesodermal proliferation have been produced experimentally in the monkey (Macaca mulatta) by Richter (1949). Investigating the effect of certain quinoline compounds, notably substances belonging to the group of 8-amino-quinolines, on the nervous system of the macaque, he found the symptoms and pathological changes so strikingly constant and extreme as to merit a full description. In brief, these changes consisted of a proliferation of capillaries and pre-capillaries within areas of parenchymal destruction. Within the lesion the cell bodies of the neurons, and the interstitial tissue including the oligodendroglia had undergone virtually complete dissolution. The microglia was more resistant and was actively proliferated, both within the core of the lesion and more markedly at the periphery. The selectivity of the focal lesions was remarkable : the thalamus, hypothalamus, red nucleus, substantia nigra, oculo-motor and trochlear nuclei, abducens, vestibular, cochlear, granule and cuneate nuclei, lateral nucleus of medulla, and the reticular formations of the pons and medulla were all affected. Richter concluded that

" a number of compounds built upon the quinoline nucleus have a peculiarly destructive affinity for the central nervous system depending upon the nature and arrangement of their side chains. In part this toxic action is expressed in relatively selective focal necroses, notably of brain stem nuclei, sensory and motor."

In the subsequent discussion, however, DennyBrown commented on the "very close resemblance between these lesions and those of Wernicke's encephalopathy" and considered it possible that these substances had an anti-thiamin effect.

Whatever the mechanism, there is no doubt of the very close similarity between these experimental lesions and the findings in the case reported here. Both are fascinating examples of the phenomenon of selective localization in the nervous system. Whether, in this infant, an unknown toxin or virus was responsible for these lesions, or whether the case was primarily a Wernicke's encephalopathy in an infant, is difficult to decide. The clinical history and course do not help greatly in reaching a decision, but are slightly more in favour of a deficiency disorder than an infective or toxic process. Owing to our scant knowledge of early infantile disease processes, it will be an advantage to keep an open mind on the aetiological problem.

\section{Summary}

A case is reported of a male infant aged 7 months dying within a period of six weeks from a central nervous system disorder characterized by somnolence, blindness, deafness, and spasticity of the limbs.

The neuropathological findings consisted of focal, bilaterally symmetrical subacute necrotic lesions or softenings extending from the thalamus to the pons, the inferior olives, and the posterior columns of the spinal cord.

Histologically the affected areas showed an intense vascular, microglial and histiocytic proliferation, and a less marked astrocytic increase. There was severe neuronal damage in these areas.

The aetiology and pathogenesis of the condition is discussed.

My thanks are due to Professor H. A. Magnus, of King's College Hospital, who supplied the brain. Professor Alfred Meyer has been a constant stimulus throughout, and I am indebted to Professor Dorothy Russell for her opinion on this case.

\section{REFERENCES}

Adams, R. D., Cammermeyer, J., and Denny-Brown, D. (1949). J. Neuropath. exp. Neurol., 8, 1.

Bender, L., and Schilder, P. (1933). Arch. Neurol. Psychiat., Chicago, 29, 990.

Ferraro, A., and Roizin, L. (1942). J. Neuropath. exp. Neurol., 1, 81 .

Greenfield, J. G., and Turner, J. W. A. (1939). Brain, 62, 227.

Meyer, A. (1944), Journal of Neurology, Neurosurgery and Psychiatry, 7, 66.

Richter, R. (1949). J. Neuropath. exp. Neurol., 8, 155.

Spillane, J. D. (1947). "Nutritional Disorders of the Nervous System." Edinburgh.

Wernicke, C. (1881). "Lehrbuch der Gehirnkrankheiten." Vol. 2, p. 229. Berlin.

Wyburn-Mason, R. (1943). "The Vascular Abnormalities and Tumours of the Spinal Cord and its Membranes." London.

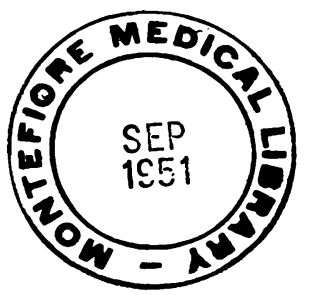

